\title{
On the front-end design of mid-frequency aperture array for square kilometre array
}

\section{Y. Zhang ${ }^{1}$ - A. El-Makadema ${ }^{1}$ - E. de Lera Acedo ${ }^{2}$ - P. Benthem ${ }^{3} \cdot$ S. Barth . $^{4}$} A. K. Brown ${ }^{1}$

Received: 5 June 2018 / Accepted: 20 September 2018 / Published online: 26 October 2018

(C) The Author(s) 2018

\begin{abstract}
Complementary to the conventional dish radio telescopes, aperture arrays provide a technically attractive approach to achieve a large field of view and flexibility in observational parameters e.g. Sky area vs. bandwidth. Designs of both aperture array elements and overall geometry for the SKA Mid Frequency Aperture Array are presented here, together with resulting trade-offs. The paper reports the latest developments of global efforts on the front-end design of Mid-Frequency Aperture Array, not attempting to make technology selections, as the priority of sciences and the time for implementing Mid-Frequency Aperture Array is yet to be fully confirmed. Different on-going front-end solutions are introduced, particularly crossed ring antenna array with a planar structure is explored in more detail as it is less known in the community. Key performances of the candidate front-end technologies are addressed by examining the prototypes. The objective of the collaborative study is to increase technology readiness for implementation of Mid-Frequency Aperture Array in the future.
\end{abstract}

Keywords Aperture array $\cdot$ Phased array $\cdot$ Polarization $\cdot$ SKA

Y. Zhang

david.zhang@manchester.ac.uk

1 School of Electrical and Electronic Engineering, University of Manchester, Manchester, M13 9PL, UK

2 Cavendish Laboratory, University of Cambridge, Cambridge, CB3 0HE, UK

3 ASTRON Netherlands Institute for Radio Astronomy, Dwingeloo, NL-7991 PD, The Netherlands

4 Observatoire de Paris, Station de Radioastronomie de Nançay, Nançay, 18330, France 


\section{Introduction}

The Square Kilometre Array (SKA) has origins pre 1990 when a number of authors published work arguing the need to construct a high sensitivity instrument to detect Neutral Hydrogen(HI) at extreme redshifts. On 8 October 1990, the IAU colloquium 131 was held in Soccorro, New Mexixo, USA. At this meeting, Wilkinson [1] presented the science case for a radio telescope with 1 square kilometre collection area to detect $\mathrm{HI}$ in galaxies at high redshift. Dish antennas are preferred in this work to form the large collection area which is essential to obtain the sensitivity and the angular resolution required. Prior to this meeting, Jan E. Noordam, Robert Braun, and A. G. de Bruyn had proposed a Dutch Neutral Hydrogen telescope [2] between 1988 and 1990. At about the same time, Govind Swarup was also proposing a next generation centimetre radio telescope [3] based on then on-going Giant Metre Radio Telescope [4] (GMRT) project. Parijsky mentioned the new century radio telescope [5] to meet the challenges of the exponential growth of the electromagnetic pollution by our civilization in 1992 .

The classical noise signal characteristics from sky show a frequency dependence such that above several hundred $\mathrm{MHz}$ the total noise at the receiver is dominated by self-generated noise in the front-end components of the receiving system. This noise level is further complicated by increasing Radio Frequency Interference (RFI). SKA with a low noise temperature and a high dynamic range has to overcome these challenges with sufficient sensitivity to resolve the invisible sky in the early Universe. This results in a requirement for a large effective area instrument. SKA surveys also require large effective Field of View (FoV) so that survey speed can be maximized, enabling practical all sky surveys [6].

It has always been the primary goal of SKA to study faint spectral line emission from the early Universe [7-9]. SKA will be the premier imaging machine [10] in the world with its milliarcsecond-scale angular resolution and sensitivity of $2 \times 10^{4} \mathrm{~m}^{2} / \mathrm{K}$. The wide field of view and high sensitivity make the SKA a superior instrument to chart distribution of galaxies and the matter structure of the Universe to greater depths on large scales. Expected uses of the HI from SKA has being changing over time. Pulsar research [11-13] has gained importance and further stimulated by gravitational wave detection such as LIGO and LISA [14]. Transient effects are providing new information in radio astronomy $[15,16]$. However the exploration of unknown is more likely to make the most significant new discoveries [17]. It is desired for the SKA to use phased arrays at lower frequencies and dish arrays at higher frequencies [18] due to the extremely large number of elements needed at high frequencies which is unlikely to be affordable.

Traditionally, the cost of such arrays at microwave frequencies has limited their application. Recent large scale integrated circuit development now allows cheap duplication of low noise receivers attached to many individual receptors. It is now possible to provide an extremely large collection area by using arrays of smaller elements providing electronically steered multiple beams and wide frequency bandwidth.

The broadband aperture array with multibeam techniques significantly increases the effective FoV (approximately two orders of magnitude than other existing 
telescopes) and this allows observing a large fractions of the sky in a short period of time with a very wide bandwidth. These unparalleled properties of aperture array are critically beneficiary also extremely important for transient sciences [19], particularly Fast Radio Bursts(FRBs). More discovery of the FRBs will help to understand the dark energy equation of state. The potential FoV of several hundred square degrees of Mid-Frequency Aperture Array (MFAA) makes it a prime candidate for discovering FRBs in sufficient numbers to do world-class cosmology, and effectively a 10-gigapixel ultra-wide field spectroscopic camera.

One of the milestones in using wide band phased arrays for astronomical observation was the THousand Element Array (THEA) in 2000 [20]. Currently, the developments of LOw Frequency ARray(LOFAR) [21], the Electronic Multibeam Radio Astronomy Concept (EMBRACE) [22] and The Murchison Widefield Array (MWA) [23] have worked as effective pathfinders to provide valuable experiences to design and implement the planned aperture array in SKA. The deployment of SKA is scheduled in two phases. The Low Frequency Aperture Array (LFAA) [24] with $2^{17}$ elements in 512 stations operating between $50 \mathrm{MHz}$ and $350 \mathrm{MHz}$, a major part of SKA phase 1, is a sparse irregular array. The development of the more technically challenging MFAA system is envisaged in SKA Phase 2 commencing in the early 2020s. The MFAA is conceptually considered technically and astronomically less mature than other conventional receiving technologies. However they represent the most competent technology available with its wide field polarimetric capabilities and the ability to detect very small variations in the observed signal.

The MFAA is particularly well suited to perform the neutral hydrogen 'billion galaxy survey'. The high level design parameters, derived from science requirements [25] for SKA MFAA, are summarized in Table 1. There is a large engineering and science challenge to implement MFAA within the cost, power and timescale constraints of SKA [26, 27]. This work discusses the technical challenges and the solutions in connection with the latest developments on MFAA in the SKA community globally, in particular the front-end design which dominate the receiver noise. In addition,

Table 1 SKA MFAA high level design requirements

\begin{tabular}{|c|c|c|}
\hline Parameter & Goal & Main sciences that drive the requirements \\
\hline Low Frequency (MHz) & 400 & High redshift HI, Steep spectrum sources \\
\hline High Frequency $(\mathrm{MHz})$ & 1450 & HI, continuum applications, Transients \\
\hline Sensitivity at zenith $\left(\mathrm{m}^{2} / \mathrm{K}\right)$ & 10000 & HI continuum deep fields \\
\hline Field of View $\left(\mathrm{deg}^{2}\right)$ & 200 & Transients \\
\hline $\begin{array}{l}\text { Maximum angular resolu- } \\
\text { tion }(\operatorname{arcsec})\end{array}$ & 0.5 & Continuum surveys \\
\hline Scan angle from zenith (deg) & 45 & Cosmology; transients; pulsar searches; Galactic HI \\
\hline Overall sky coverage $\left(\mathrm{deg}^{2}\right)$ & 25000 & Cosmology; transients; pulsar searches \\
\hline Polarization purity $(\mathrm{dB})$ & $\begin{array}{l}-25 \text { (off } \\
\text { axis) }-40 \text { (on } \\
\text { axis) }\end{array}$ & Pulsar timing ; cosmic magnetism \\
\hline Spectral Dynamic Range (dB) & 60 & $\mathrm{HI}$; BAO and galaxy evolution \\
\hline
\end{tabular}


the total number of elements required is determined by the chosen array geometry and it has a direct cost and power implications on closely connected or associated electronics. Hence two typical array geometry configurations are also investigated.

\section{Aperture array antennas}

Aperture Arrays have been progressively developed to become a strong candidate technology for radio telescopes capable of a large collecting area. The cost of an element is critical for SKA implementation since the total number of elements is on the order of millions. The antenna element needs to operate between two and three octaves in frequency bandwidth. To allow the Stokes Parameters of the received field to be measured two orthogonal polarization components are needed over the entire bandwidth. Additionally a wide scan angle must be provided to achieve a wide FoV. Low noise is another parameter critical for providing the high sensitivity required in radio astronomy applications, as a result, the low-loss antenna elements and low noise receivers have been desired. Multiple broadband antenna element designs for aperture array application are examined in the following sections. In particular, Crossed Octagonal Ring Antenna (C-ORA) is covered more intensively as it represents a novel approach compared to other antennas and less known in the community. However, other antennas mentioned below have being continuously improved and actively used for radio astronomy, it is beneficial to report their progress and performance as a benchmark. An artistic view of a MFAA station based on C-ORA and a section of C-ORA array is shown in Fig. 1.

\subsection{Tapered slot antenna}

Tapered Slot Antenna (TSA) was proposed by Lewis in 1974 [28].TSA Antennas are widely utilized in mobile telecommunications and radars. One type of TSA whose slot is opened exponentially and it was called Vivaldi antenna [29]. Vivaldi Notch antennas can provide bandwidths up to several octaves in phased arrays that scan over wide angles. A parameter study of Vivaldi Notch-Antenna for phased array is investigated in [30]. Within ASTRON multiple demonstrators and phased array
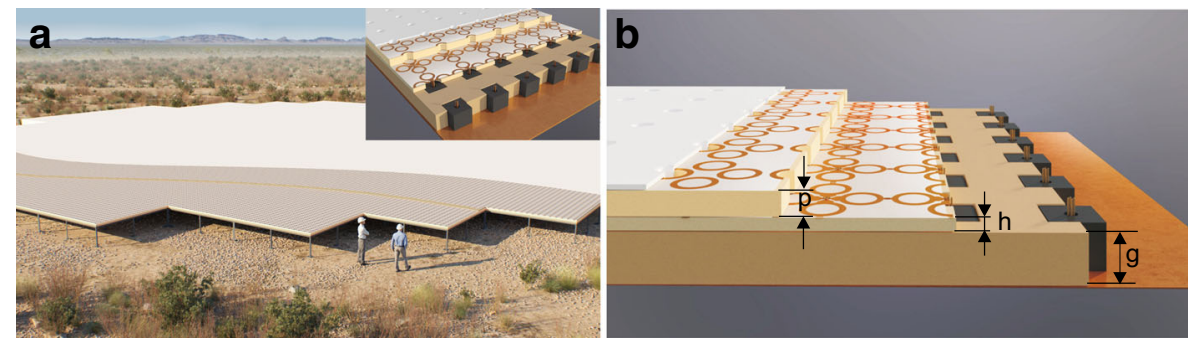

Fig. 1 Mid-frequency aperture array based on crossed octagonal ring antenna 
instruments have been built. The THousand Element Array (THEA) is the first outdoor phased-array system used to detect (strong) radio sources using adaptive digital multi-beam beamforming techniques employing Vivaldi antenna [20]. The total number of Vivaldi antenna elements in THEA is 1024. EMBRACE is a dual station single polarization aperture array telescope, which covered an area of $162 \mathrm{~m}^{2}$ in Westerbork, The Netherlands and $70.8 \mathrm{~m}^{2}$ in Nançay, France. EMBRACE in Westerbork has 10368 active elements and 4608 active Vivaldi antenna elements are used in Nançay. The performance of the Vivaldi antenna designed for EMBRACE is described in [22]. Two EMBRACE stations have been tested in different phases of their construction [31-33].

EMBRACE at Nançay has been fully operational since 2011. Characterization of the $70.8 \mathrm{~m}^{2}$ dense array at Nançay is reported in $[34,35]$. APERTIF, a focal plane array instrument, currently being commissioned, uses 12 dishes of the Westerbork Synthesis Radio Telescope [36]. Each focal plane array holds 121 Vivaldi elements. Most recently the Low Noise Tile prototypes have been built to further decrease the receiver noise figure of the antenna and LNA combination to $35 \mathrm{~K}$ levels [37] and below at room temperature. Environmental prototypes are installed at the SKA Karoo site to gain experience in material degradation, tile designs and local rodents. The new mechanical connections have been made for Vivaldi antennas for SKA MFAA [38] to ensure resistance to damage from local rodents. A prototype tile of Vivaldi antenna array is shown in Fig. 2.

\subsection{Crossed octagonal ring antenna}

The initial Octagonal Ring Antenna (ORA) is a novel dual-polarized array antenna with broad bandwidth. The detailed ORA design has been described in [39]. Very different to Vivaldi antenna design, the ORA is designed to be a planar, easily fabricated, and potentially low cost structure. The ORA operates at wide scan angles with a smooth polarimetric performance over the entire FoV. The fundamental electromagnetics has been confirmed through previous studies with field measurements

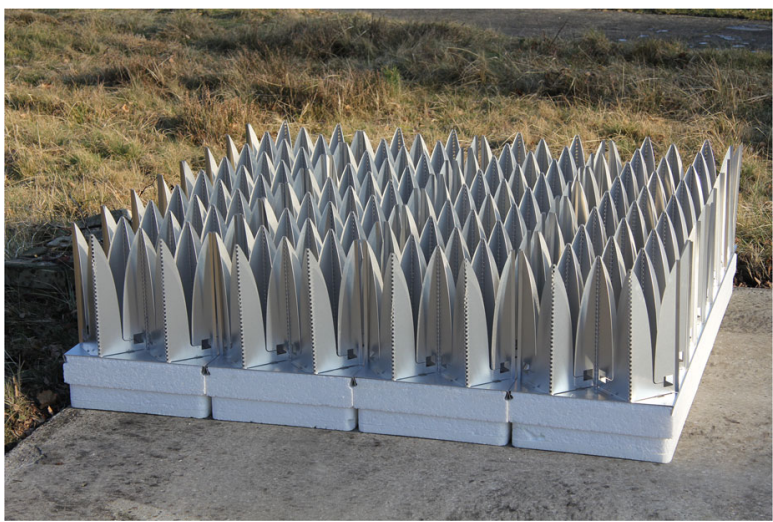

Fig. 2 The Vivaldi antenna array tile for SKA MFAA, developed in ASTRON, The Netherlands 
[39]. In order to feed elements of dual polarization at the same position, hence a Low Noise Amplifier (LNA) board for two polarizations can be shared, Crossed Octagonal Ring Antenna (C-ORA) is proposed based on the same principle as ORA. The layered antenna array structure and the artistic view of the corresponding MFAA station is shown in Fig. 1.

The array based on C-ORA elements is a three-layered structure. The embedded active radiators or receptors are mutual coupled rings with a meta-material superstrate layer above the element rings. The embedded receptors and the active layer they formed are above the groundplane with a defined distance. The unit cell design of C-ORA element is illustrated in Fig. 3. The dual polarized elements are fed at the crossing point and share the same phase center. To achieve the required bandwidth this antenna includes capacitive coupling loads between the elements. The C-ORA structure itself presents a high radiation efficiency leading to a low noise temperature due to its unique configuration. In addition, a customized balanced LNA using a Monolithic Microwave Integrated Circuit (MMIC) has been produced and integrated locally with the antenna element to minimize feeding loss [40]. This work is ongoing. With the current LNA design, the view of the LNA box and its respective location to the antenna elements is shown in Fig. 1b.

To reduce the number of active elements needed in the aperture, the C-ORA design is also being explored with non-Cartesian distribution of the elements. This new geometry is not viable from the conventional Vivaldi-type antennas. A triangular grid based C-ORA array is designed and the prototype has been produced and compared to a more conventional square grid C-ORA. Unit cells of the C-ORA designs for the

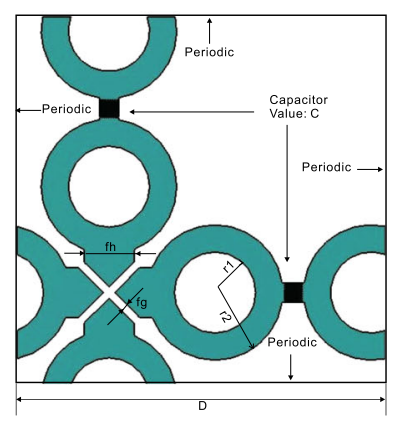

a

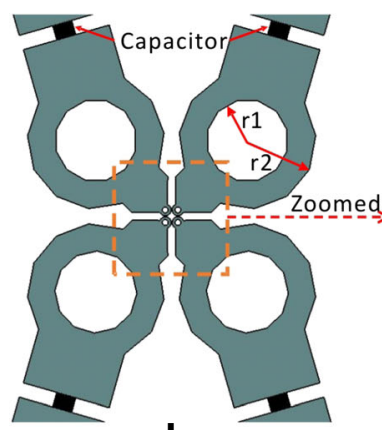

b

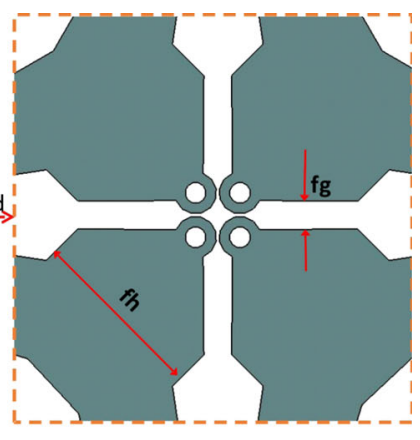

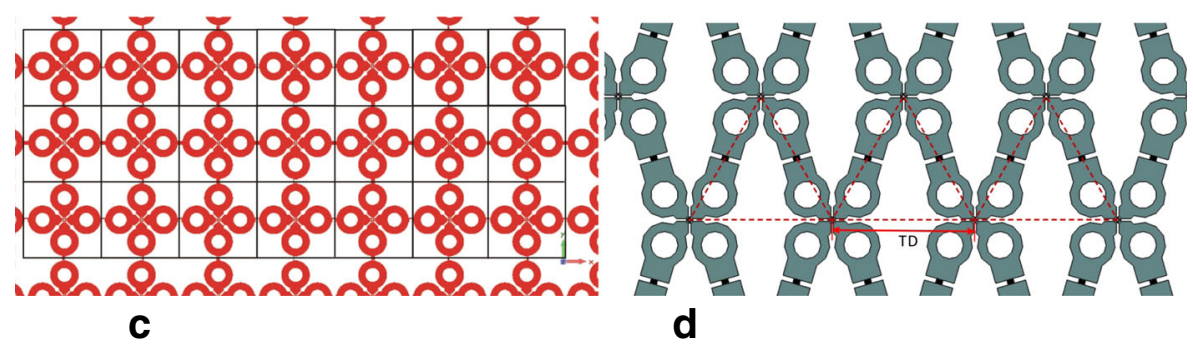

Fig. 3 The C-ORA array element design models 
square and triangular grid arrays and the respective section of arrays showing the arrangements of elements is given in Fig. 3. The value of parameters for an optimized C-ORA design is summarized in Table 2. The capacitor value between the square grid element is $0.8 \mathrm{pF}$, it can be realized by 17 inter-digitated fingers, with the length of each finger of $5 \mathrm{~mm}$ and the gap between fingers of $0.2 \mathrm{~mm}$. For the triangular grid array, the capacitor value between the element is $1 \mathrm{pF}$, it can be realized with 17 fingers, the length of each finger of $6 \mathrm{~mm}$ and the gap between fingers of $0.2 \mathrm{~mm}$.

The reflection coefficients for the C-ORA elements in an infinite array environment are shown in Fig. 4. The results are from simulations based on the optimized C-ORA designs with the parameter values given in Table 2. The performances for the C-ORA arrays with elements configured in both a square and triangular lattice are shown. They illustrate the main characteristics of the C-ORA design: providing a large frequency bandwidth $(400 \mathrm{MHz}$ to $1.45 \mathrm{GHz})$; operating over wide scan angles $\left( \pm 45^{\circ}\right.$ from the zenith). It indicates that the aperture array based on C-ORA receptors of a planar structure has the ability for simultaneous measurements over large fractions of the sky (optical FoV over $200 \mathrm{deg}^{2}$ ) at the mid-frequency band of SKA.

The same number of elements were used in both configurations of the finite array prototypes, so that the triangular grid array occupies a larger physical area than the square grid. Accordingly it is expected that the gain and hence sensitivity of the triangular grid array is higher than the square grid using the same number of elements. This is reflected in a narrower beam width for the triangular grid. Initial measurements are given in Fig. 5, which confirms the simulation results. It is stressed these are early measurements and do have significant measurement error due to site effects but nonetheless indicate the triangular grid approach is worthy of further investigation and refinement.

It reveals that approximately $13 \%$ total number of elements can be saved to achieve the same sensitivity with a triangular grid based C-ORA array. It is noted that the

Table 2 Parameters for C-ORA design

\begin{tabular}{lll}
\hline Parameter & Value $(\mathrm{mm})$ & Description \\
\hline Square grid design & & \\
D & 105 & Element separation \\
r1 & 12 & Radius for inner circle \\
r2 & 20 & Radius for out circle \\
fh & 15 & Width for feeding strip \\
fg & 2.8 & Gap for feeding strip \\
Triangular grid design & & \\
TD & 121.24 & \\
r1 & 12 & Length of the equilateral \\
r2 & 20 & Radius for inner circle \\
fh & 16 & Radius for out circle \\
fg & 2.5 & Width for feeding strip \\
\hline
\end{tabular}



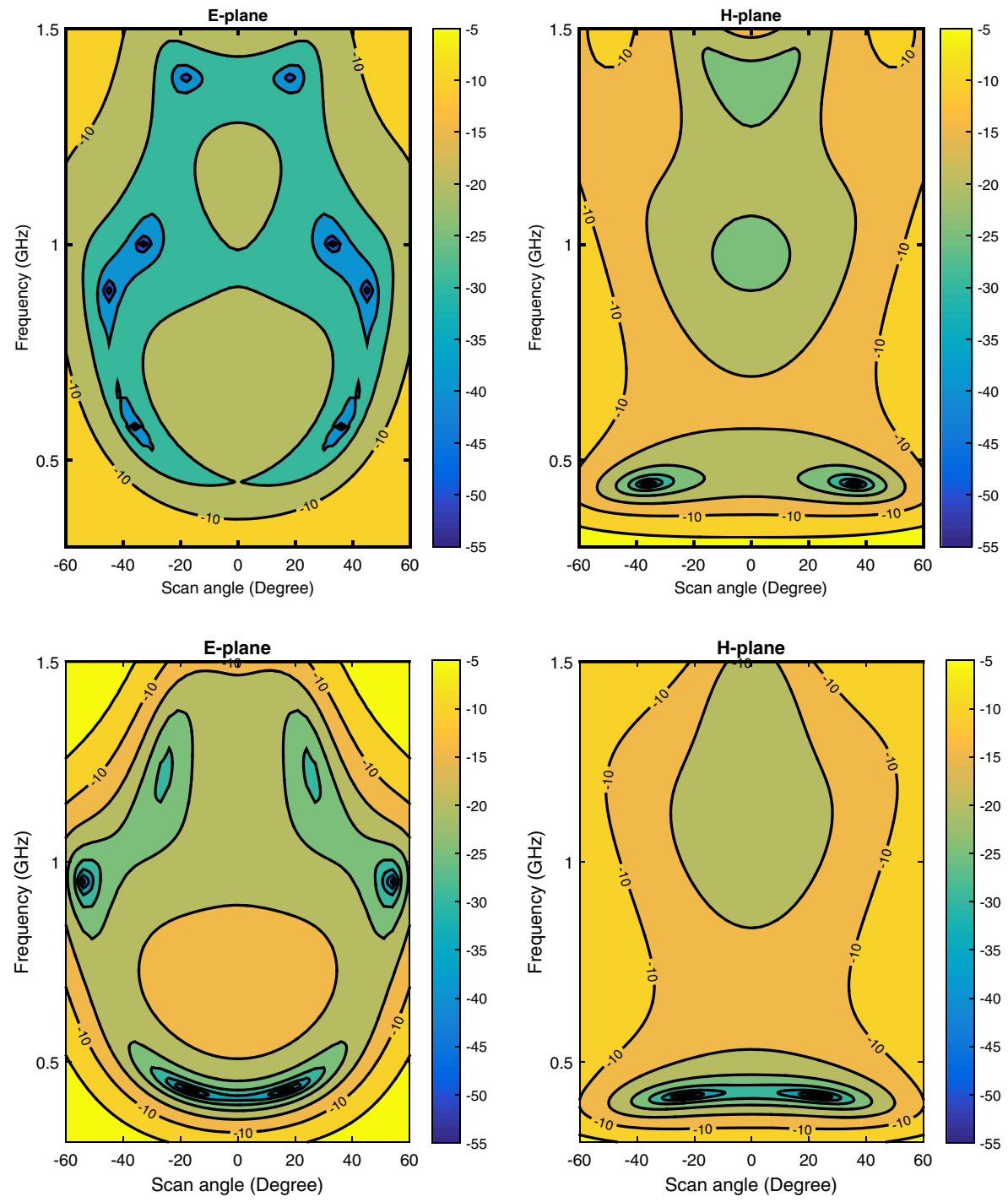

Fig. 4 The simulated active reflection coefficients in $\mathrm{dB}$ of C-ORA elements in infinite arrays, the arrays are scanned to wide angles

sidelobes for the triangular grid based array has no significant increase compared with that of the square based array even the element separation is greater. However, a 5-10 dB rise in cross polarization for the triangular grid array is observed compared to the square grid array. The triangular grid allows for larger separation between elements compared to a square grid without effecting the maximum scan performance limited by the occurrence of grating lobes. This makes it possible for the triangular grid to cover the same aperture area as the square grid but with less elements. 


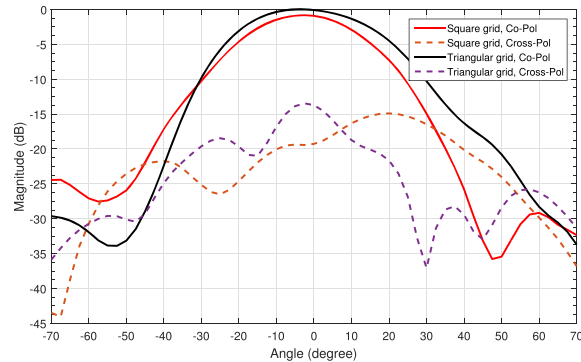

(a) $900 \mathrm{MHz}$.

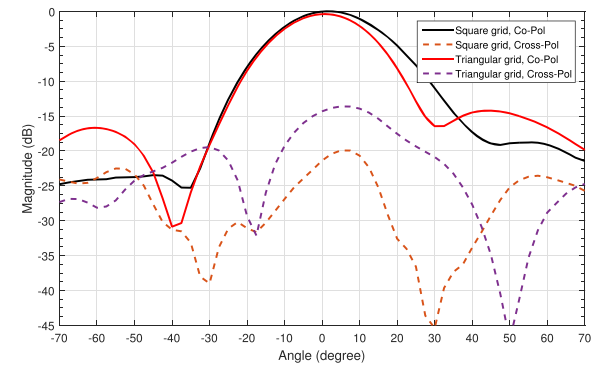

(b) $1200 \mathrm{MHz}$.

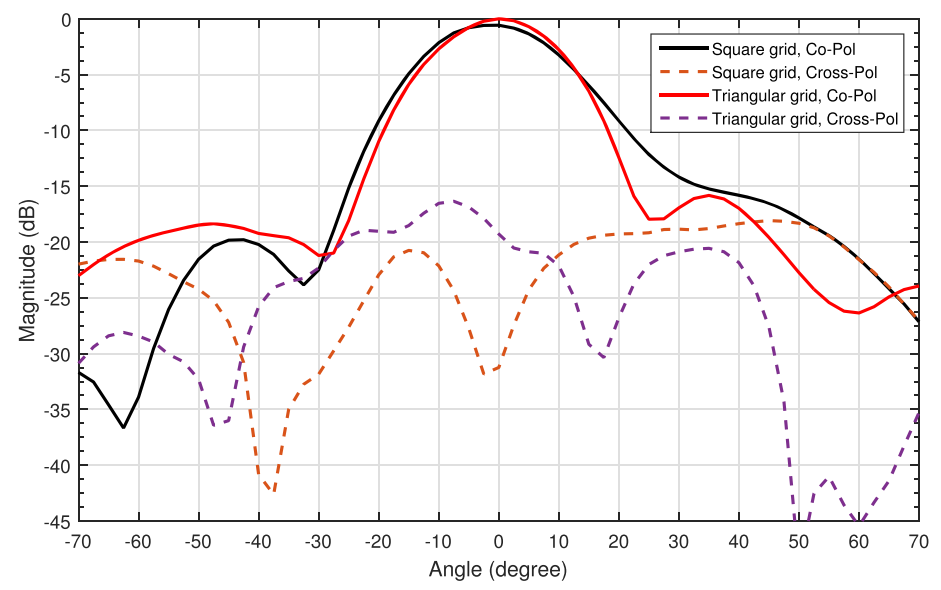

(c) $1400 \mathrm{MHz}$.

Fig. 5 The C-ORA array based on square and triangular grid, measured radiation patterns of the $4 \times 4$ subarrays in the diagonal plane (D-plane) for the bore-sight reception

\subsection{Log periodic dipole array}

The Log Periodic Dipole Array (LPDA) for MFAA is essentially a progression of coplanar dipoles that increase size logarithmically as defined by a constant ratio. It is derived from the LPDA concept for SKA-LFAA [41]. Different to the Vivaldi and C-ORA mentioned above where the mutual coupling between the adjacent elements are effectively employed to yield the frequency bandwidth, the single LPDA element is designed to operate over the entire MFAA frequency band for sparse array geometry [42] and using a single ended LNA in the current version. This would deliver reduced power consumption with respect to a differential LNA (this may be important considering the antenna numbers for MFAA) and is relatively feasible for the MFAA bandwidth ratio - aprox. 3:1. A detailed trade-off study of matching/bandwidth/linearity between the differential option (typically more suited for wide bandwidths and better common mode rejection) and the single ended option will be done in the coming years. The small profile is sought to reduce the footprint of the antenna, and hence allow a higher frequency sampling if needed. 
Table 3 Parameters for LPDA design

\begin{tabular}{lll}
\hline Parameter & Value & Description \\
\hline$\alpha$ & $62.5^{\circ}$ & Outer aperture of the teeth \\
$\beta$ & $3^{\circ}$ & Inner aperture of the teeth \\
$w_{1}$ & $37.5 \mathrm{~cm}$ & Antenna width \\
$w_{2}$ & $28.5 \mathrm{~cm}$ & Log-periodic progression width \\
$h_{1}$ & $30 \mathrm{~cm}$ & Antenna height \\
$h_{2}$ & $5 \mathrm{~cm}$ & Distance from the last dipole to the GND plane \\
$h_{3}$ & $7.1 \mathrm{~cm}$ & Bowtie bottom dipole \\
$\delta$ & $45^{\circ}$ & Bowtie dipole angle \\
\hline
\end{tabular}

The current prototype design has 6 dipoles and its shape has been optimized using simulations in order to remain effective at the low end of the frequency band. It is noted that the overall element size is significantly smaller through iteration of design studies. The size of the current element model is $37.5 \mathrm{~cm}(\mathrm{~W}) \times 37.5 \mathrm{~cm}(\mathrm{~L}) \times 30 \mathrm{~cm}$ (H). The footprint of the LPDA for MFAA can be reduced further to allow the transition between the dense and sparse regimes, particularly at the high frequency. The main parameters for LPDA design is given in Table 3. Initial measurements on a 16 element LPDA array (1 tile) for the MFAA band with a sparse random configuration have been conducted at the Mullard Radio Astronomy Observatory, south of Cambridge, UK. The measured results on scattering parameters have a good agreement with the electromagnetic modeling, allowing further optimizations and characterizations for a greater scale investigation [43]. A larger array with 8 tiles, optical fiber output for the antennas and digital beam forming boards is currently been developed at Cambridge to continue the studies on the sparse array design and calibration. Furthermore, in collaboration with Cambridge Consultants Ltd., a mass production version of this antenna is being developed in order to reduce the unit cost and improve the life time of the design. The design model and the 16 element prototype tile is illustrated in Fig. 6.

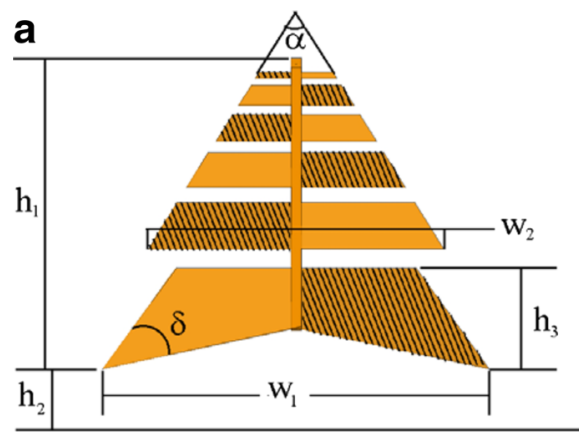

b

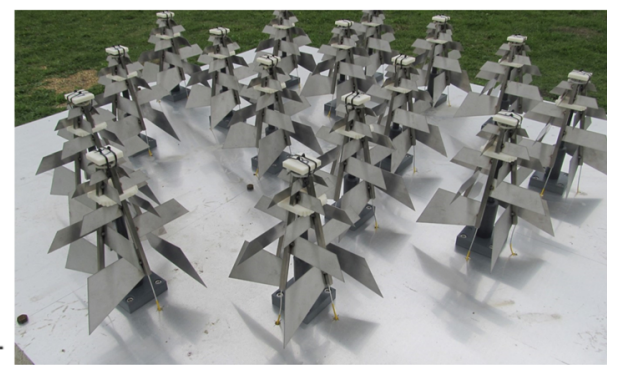

Fig. 6 The LPDA designed for SKA MFAA at University of Cambridge 


\section{Array geometry}

The high sensitivity for SKA telescope as mentioned in Table 1 demands a large number of wideband antenna elements in the aperture array. The array geometry regarding the arrangement and spacing for these elements has a significant effect on the array performance at different frequencies over the entire band [44]. The prime considerations linked to the array geometry are Dense/Sparse regimes and regular/Irregular formation of the array. A dense array samples the incoming wavefront at least at the Nyquist rate by having elements spaced $\leqslant \lambda / 2$. As the frequency reduces the array oversamples the wavefront resulting in the effective aperture $\left(A_{\text {eff }}\right)$ remaining roughly constant with frequencies. The benefit of this fully sampled system is that there can be very tight control of the beams. This type of array has the highest dynamic range capability of AAs. Spectral dynamic range is defined as the ratio of peak brightness to the residual instrumental spectral response, $\mathrm{I} / \Delta \mathrm{I}$, at the source location over a specified spectral interval. However, for a given number of elements, a dense regular array will suffer from a loss of sensitivity at low frequencies where the physical aperture is oversampled and furthermore the effects of mutual coupling are harder to deal with compared to sparse irregular (random) arrays.

A sparse array, as its name implies, has elements spaced further apart than $\lambda / 2$. In the limit, each element can act independently and provide an element level $A_{\text {eff }}$ which scales as $\lambda^{2}$. This is of benefit particularly at the lower part of the frequency band where sky noise becomes dominant. The increasing $A_{\text {eff }}$ towards low frequency increases the sensitivity and hence survey speed which helps counteract the decreasing extragalactic flux density from the sources. The sensitivity of a telescope is defined as [45]

$$
S=\frac{A_{e f f}}{T_{s y s}}
$$

Where $A_{e f f}$ is the effective area of the telescope and $T_{s y s}$ is the noise temperature of the whole system, $A_{\text {eff }}$ can be derived as

$$
A_{e f f}=\frac{\lambda^{2}}{4 \pi} D
$$

where $\lambda$ is the wavelength and $D$ is the directivity of the array, and the $T_{s y s}$ can be calculated by

$$
T_{s y s}=\eta_{\text {rad }} \cdot T_{s k y}+T_{0}\left(1-\eta_{\text {rad }}\right)+T_{\text {rec }}
$$

where $\eta_{\text {rad }}$ is the radiation efficiency of the antenna, $T_{s k y}$ is the sky noise temperature, $T_{0}$ is the antenna noise temperature and $T_{r e c}$ is the receiver noise temperature. The sensitivity for the MFAA telescope is shown in Fig. 7. In this calculation the antenna noise temperature over the frequency band is derived from Medellin model in [46] and the receiver temperature, $T_{r e c}$, is assumed $38 \mathrm{~K}$. $\mathrm{T}_{s k y}=1.691 \times(f)^{-2.751}$ +4.875 , and the sensitivity of the MFAA telescope based on sparse array of LPDA and dense array of C-ORA is shown in Fig. 7. It is worth noting that the frequency profile of the sparse array showing peaks and troughs is due to the directivity of the current LPDA design that has 6 dipoles to cover the entire band. This prototype is now being optimized to reduce this ripple. The criteria for the calculation is that the sensitivity of the instrument at $800 \mathrm{MHz}$ is to achieve $10000 \mathrm{~m}^{2} / \mathrm{K}$. The number of 


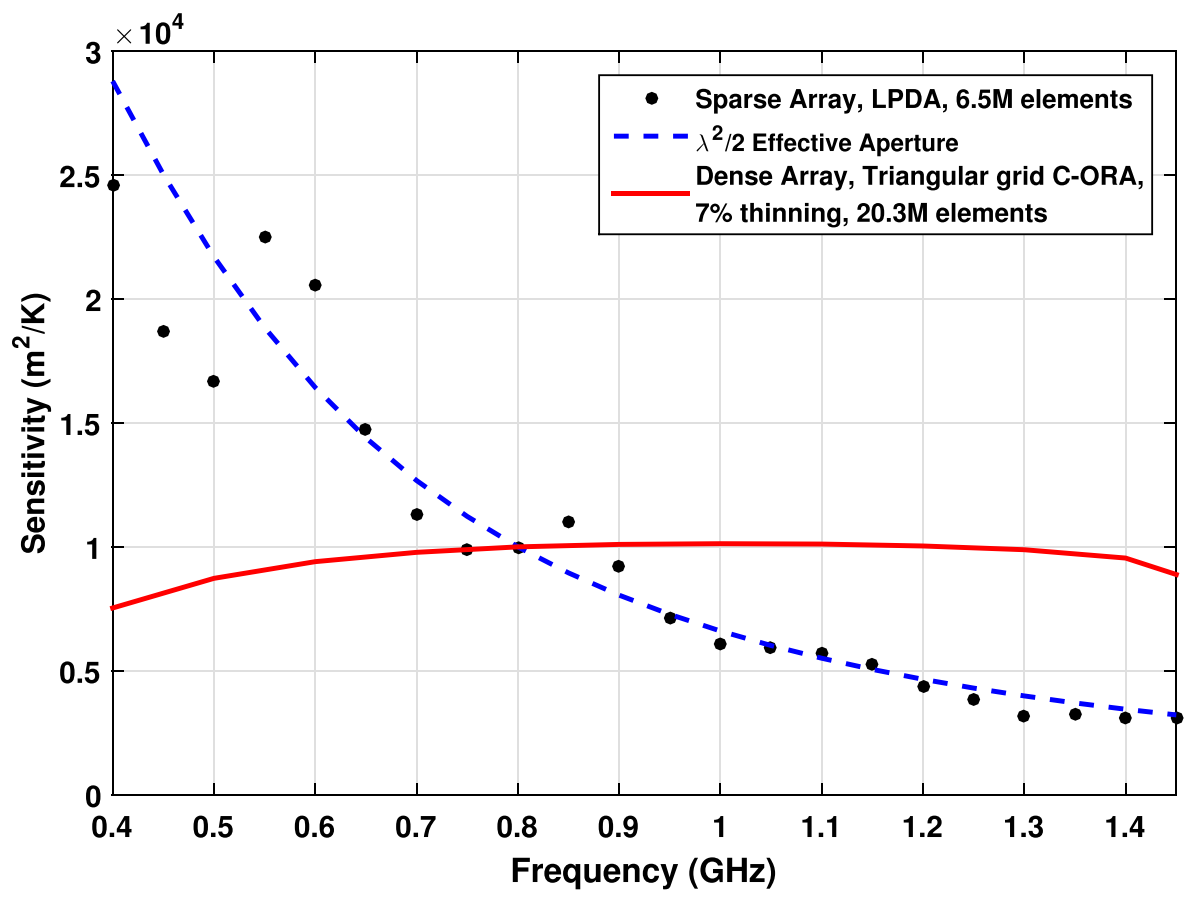

Fig. 7 The sensitivity for sparse and dense aperture arrays for MFAA, the sparse array is based on LPDA and the dense array is based on C-ORA. Both are from simulated results

elements to meet the requirement for a sparse LPDA array is 6.5M, 25.3M elements for C-ORA based on the regular square grid, and 22M elements for triangular grid based C-ORA array. Both square and triangular based C-ORA dense array can be furthered randomly thinned. C-ORA dense array based on regular triangular gird can be thinned by $7 \%$ and the total number of elements for C-ORA can be reduced to 20.3M [47].

The sparse array shows its merit at the low frequency band (below $800 \mathrm{MHz}$ ) due to the exponential increase of effective aperture towards low frequency. But for the science observations at above $800 \mathrm{MHz}$, the dense aperture array demonstrates its advantages. The sensitivity for the dense aperture array has a significant drop at the low frequency while the sparse array shows a reduced sensitivity at the high end of the frequency band. It is worth noting that the sensitivity profile shown here has ripples due to the antenna directivity for this particular design of the LPD antenna. This design is an early prototype and it can be further improved to reduce this ripple. The trade-off between dense regular arrays and irregular (or regular) sparse arrays is the key for the design of an optimum instrument for SKA MFAA since it is not only a trade-off of sensitivity at different parts of the frequency band but also there are other important considerations including cost and sidelobe control. One of the advantages of the dense sampled array over the sparse array is the sidelobe control capability. In a dense sampled array, providing the usual coupling is allowed for, then 
the sidelobes can be controlled directly by the chosen aperture weighting. In a sparse array sidelobe control is much harder with far-out sidelobes in particular can become high. Whether this is an issue or not depends on the science imaging required and, assuming each station is used in an interferometer, the geometry and effectiveness of the interferometric process in reducing residual sidelobes.

\section{Analogue front-end components}

The fully differential finite array based on C-ORA elements has been manufactured and measured in a commercial Compact Antenna Test Range (CATR). The finite array prototype and the measurement set up in the compact range is shown in Fig. 8. The array prototype $(10 \times 10$ finite array, $1.25 \mathrm{~m} \times 1.25 \mathrm{~m}$ in physical aperture size $)$ has been assembled with $8 \times 8$ elements in the centre integrated with LNAs. The radiation patterns of the $4 \times 4$ subarray with an analogue beamformer have been measured. The beamformed radiation patterns of the subarray at the centre of the finite array prototype are shown in Fig. 9. A good agreement is achieved in both $E$ - and $H$-planes at different frequencies- $900 \mathrm{MHz}, 1200 \mathrm{MHz}$ and $1400 \mathrm{MHz}$.

\subsection{Low noise amplifier}

Antenna and Low Noise Amplifier (LNA) contribute a dominant amount of noise into the whole aperture array system [48]. Their performances are the key to achieve a low system noise. The widely used technologies for LNA design are HEMTs on GaN, HBT on InP, HEMTs on GaAs and Bipolar- CMOS on silicon [49, 50]. Silicon is approximately 5 times cheaper than GaAs. SiGe technology seems to be a good compromise in the respect of requirements on cost/energy/performance. As more and more investment into silicon based technologies, Silicon Germanium
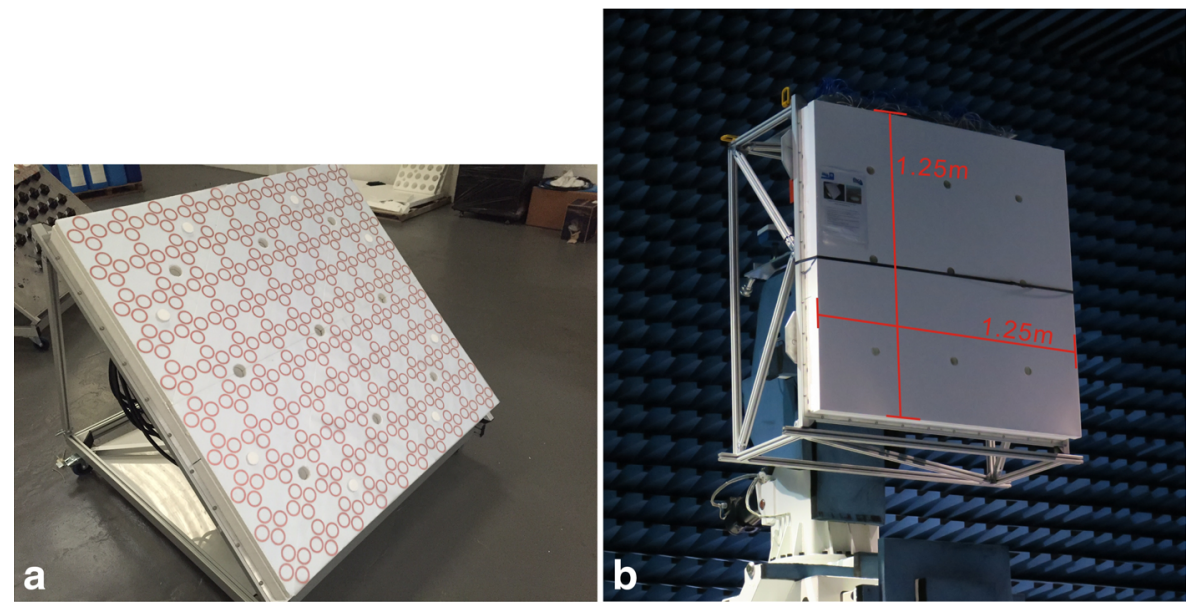

Fig. 8 The C-ORA finite array prototype and measurements of patterns in the anechoic chamber 


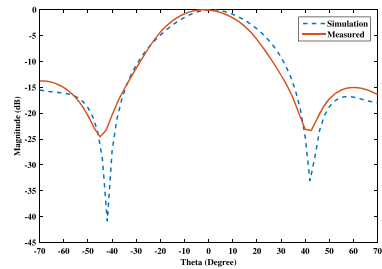

(a) E-plane, $900 \mathrm{MHz}$.

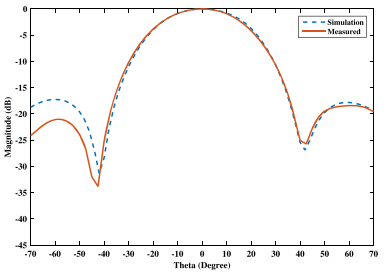

(d) H-plane, $900 \mathrm{MHz}$

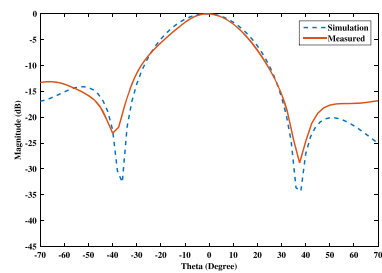

(b) E-plane, $1200 \mathrm{MHz}$.

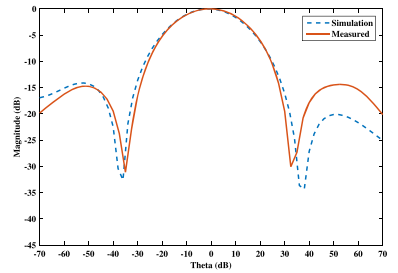

(e) H-plane, $1200 \mathrm{MHz}$.

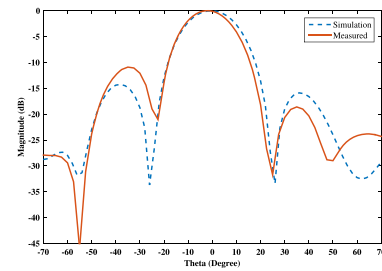

(c) E-plane, $1400 \mathrm{MHz}$.

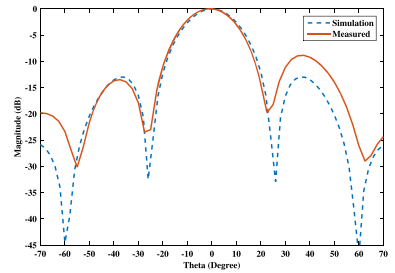

(f) H-plane, $1400 \mathrm{MHz}$

Fig. 9 The measured radiation patterns of the C-ORA subarray of $4 \times 4$ elements, an analogue beamformer was used for the broadside observation, the subarray is at the centre region of $10 \times 10$ finite array, the co-polarization patterns are shown and compared with simulations

(SiGe) heterojunction bipolar transistors (HBTs) have been continuously improved and are currently beginning to become comparative with InP HEMTs for microwave low noise amplifiers [40,51]. The latest LNA developments in the radio telescope community over the MFAA frequency band are summarized in Table 4. A MMIC differential amplifier using NXP $0.25 \mu \mathrm{m}$ QUBiC4Xi BiCMOS SiGe technology has been developed at Observatoire de Paris, Station de Radioastronomie de Nançay specifically for C-ORA design [52]. Two-stage differential cascode topology with two common-emitters in each stage is used in the design $[52,53]$. The LNA diagram, mixed-mode S-parameters of the LNA derived from measurement, and the measured noise temperature is shown in Fig. 10. The noise temperature measurement is based on the method described in [54]. Performance of the differential LNA based on BiCMOS SiGe technology under room temperature condition for the C-ORA array designed by Station de Radioastronomie de Nançay is summarized in Table 5.

\subsection{Analogue beamformer}

For cost reasons, analogue beam forming and combiners in the signal chain of the total system may be used to limit the number of digital receivers. In order to use analogue beamforming over multi octave frequency ranges it is essential to use time delay beamforming. The real time delay based analogue beamformer ASIC has been developed at Observatoire de Paris, Station de Radioastronomie de Nançay. In implementing the beamformer BiCMOS MMIC, the well-established technology from New Experience Philips (NXP) based on QUBIC4Xi platform has been used.

The QUBIC4Xi manufacturing process uses carbon enhanced SiGe base for high performance, reliable and highly integrated semiconductor devices. It minimizes the 


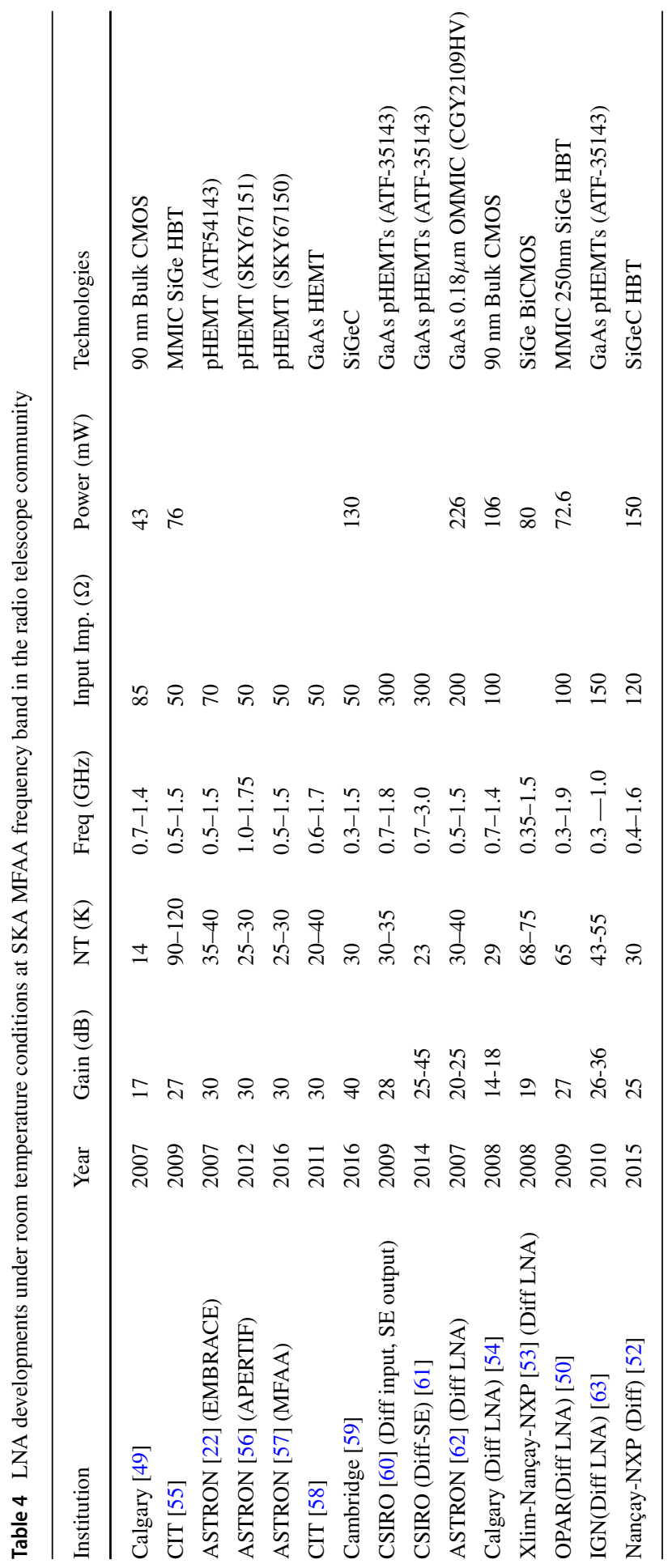




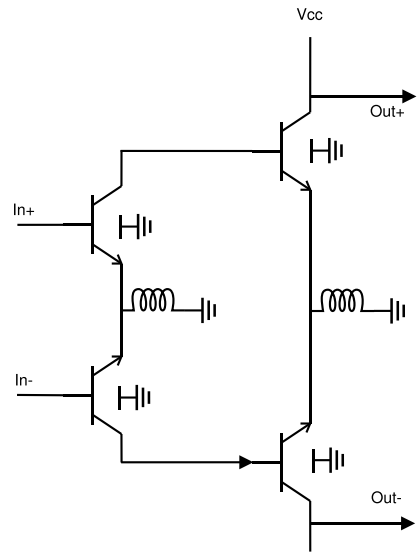

(a) Differential LNA structure

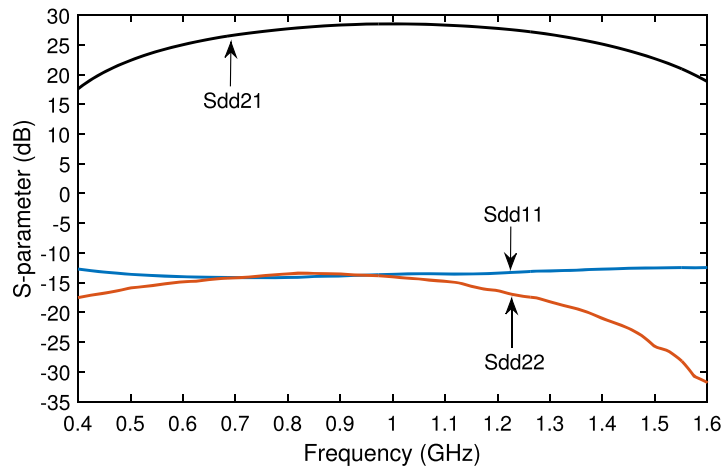

(b) S-parameters

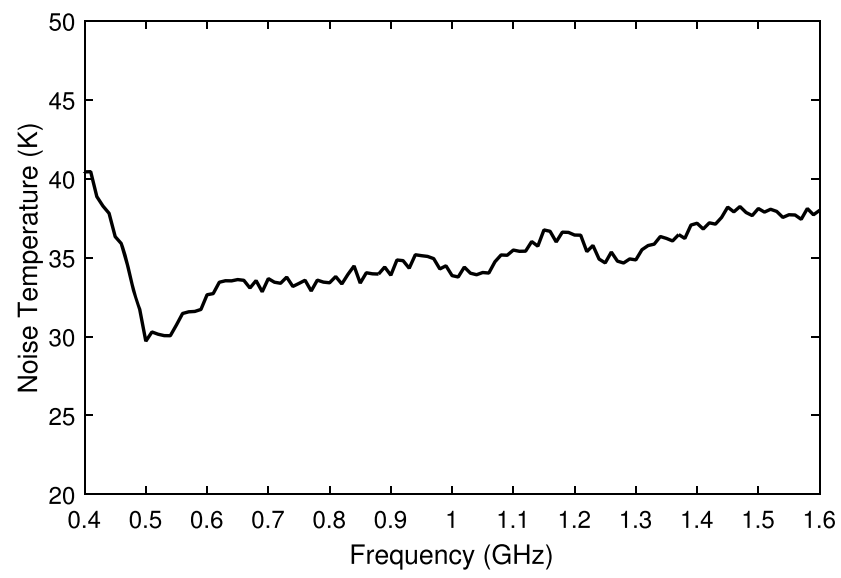

(c) Noise Temperature

Fig. 10 The diagram of the differential LNA structure and the measured performance

base resistances, while ensuring sufficient current gain and sound high frequency noise. It is based on the $0.25 \mu \mathrm{m}$ BiCMOS SiGe production process. The technology features 5 buried layers of metal interconnect, deep-trench-isolation, and double-poly HBT. A high density metal-insulator-metal (MIM) capacitors and Shielded single ended inductors are available from the library. High-Q inductors can be realized by putting metal spiral traces of a thick $\mathrm{Cu}$ above passivation layers, and using thin-film wafer-level packaging techniques. Both of them are crucial to achieve the required time delays with a minimum chip area. The size of the chip is $3.19 \mathrm{~mm} \times 3.594 \mathrm{~mm}$ including sawlanes. A simple circuit model for the delay line is the constant- $k L C$ ladder structure. The time delay of this structure is approximately $T_{d}=n \sqrt{L C}$, where $n$ is the number of $L C$ sections. The Beamformer Chip (BFC) architecture supports four input channels, and can generate two independent beams. Each channel contains its own digital control circuit to set the delay steps for the beams and a 
Table 5 Performance of the LNA based on BiCMOS SiGeC technology developed at Observatoire de Paris, Station de Radioastronomie de Nançay

\begin{tabular}{lll}
\hline Parameter & Value & Description \\
\hline $\mathrm{f}_{\text {low }}(\mathrm{MHz})$ & 400 & Low frequency \\
$\mathrm{f}_{\text {high }}(\mathrm{MHz})$ & 1600 & High frequency \\
$\mathrm{NT}(\mathrm{K})$ & 30 & Noise temperature \\
Gain $(\mathrm{dB})$ & 25 & Differential Gain \\
$V_{c c}(\mathrm{~V})$ & 3.3 & DC supply \\
$I_{B I A S}(\mathrm{~mA})$ & 46 & Current \\
OIP3 $(\mathrm{dBm})$ & 11 & Output third-order intercept point \\
Input Impedance & $2 \times 60$ ohms & Different input \\
Output Impedance & $2 \times 50$ ohms & Differential output \\
$\mathrm{P}_{1 d B}$ & $0 \mathrm{dBm}$ & $1 \mathrm{~dB}$ Compression point \\
\hline
\end{tabular}

combination network. 18 registers (bytes) have been assigned in the chip to control the delay steps and the channel gains including two registers to control the extra beam delays after combination of channels. In free space, the time delay required between two adjacent elements in the array is

$$
\Delta t=\frac{d \sin \theta}{c}
$$

where $d$ is the element separation, $c$ is the speed of light. For a $0.125 \mathrm{~m}$ element separation as in the prototype design and a $45^{\circ}$ scan angle in the principle planes, the required time delay between adjacent elements is $0.3 \mathrm{~ns}$. The manufactured MFAA prototype has $8 \times 8$ active elements where each with an integrated LNA. To achieve a combined beam requires a two-level hierarchical beamformer. In the first level, group of 4 element are combined by using one beamformer chip to form two independent beams from each group. The second level of the beamformer combines these beams also by a group of four to form a narrow analogue beam. The designed true-time delay based ASIC fully meets this requirement with a maximum $1.2 \mathrm{~ns}$ delay on each channel and an extra $1.2 \mathrm{~ns}$ delay on each beam. The measured relative time delay at different steps is shown in Fig. 11. For each channel in the beamformer chip, the amplitude can be controlled with a $0.5 \mathrm{~dB}$ step, in total 16 steps are supported. The measured gain performance of one channel in the beamformer chip is given in Fig. 12. The performance of the beamformer chip is given in Table 6 .

\section{Manufacturing process}

Design for Manufacture (DFM) techniques are essential to deliver the telescope. In order to reduce the associated transmission line losses for Vivaldi antennas, the differentially fed tapered slot antenna elements can be screen printed on a polyester foil using silver ink and have been copper plated afterwards [64], however, this presents challenges for good inter-tile connections. There is ongoing development in 


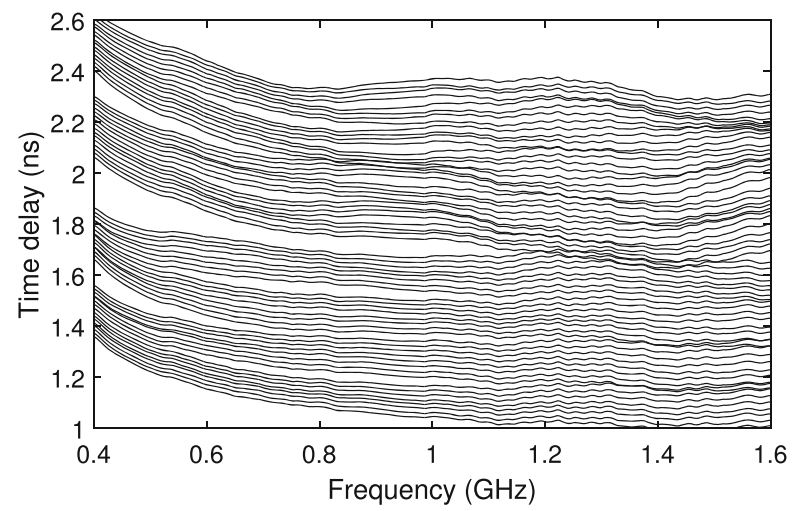

Fig. 11 The measured 64 time delay steps of one channel in the beamformer chip

Vivaldi design [38]. Compared to other conventional antenna designs, array structure of C-ORA can be fabricated by etching, ink-jet or screen printing, ideal for a large-scale low-cost implementation. The C-ORA demonstrates a robust repeatability for mass production. Several manufacturing processes have been used for the active and passive layers of C-ORA, including etching, printing and plating. At the MFAA frequency for SKA, the manufacturing techniques has no significant effect on the array performance. Recent development of Graphene ink for antenna manufacturing is investigated in [65], it provides an alternative low cost route for future mass production although the material loss is of concern using the current techniques.

\section{Prototype fabrication and test}

The active layer of C-ORA finite array is manufactured by chemical etching on N9000 PTFE laminate with the thickness of $0.127 \mathrm{~mm}\left(\epsilon_{r}=2.2, \tan _{d}=0.0009\right)$, and 0.5 ounce copper. The passive layer is etched on polyester with the thickness of

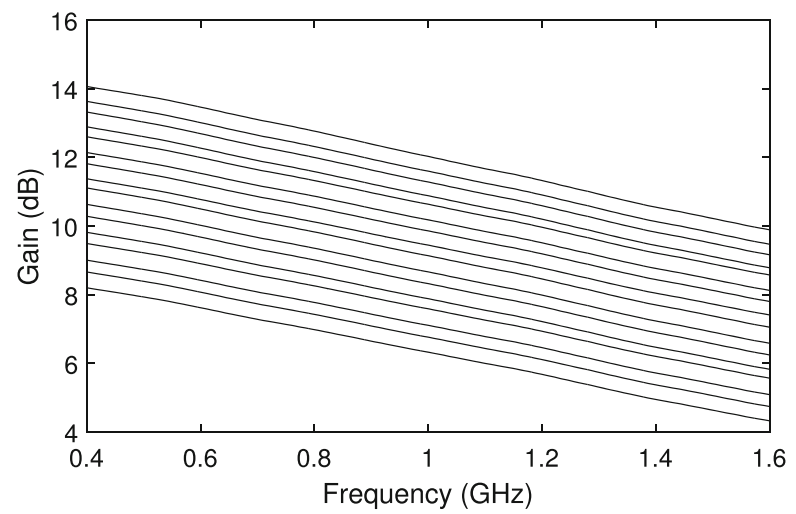

Fig. 12 The measured 16 steps of gain control of one channel in the beamformer chip 
Table 6 Performance of the time-delay based beamformer chip developed at Observatoire de Paris, Station de Radioastronomie de Nançay

\begin{tabular}{lll}
\hline Parameter & Value & Description \\
\hline $\mathrm{f}_{\text {low }}(\mathrm{MHz})$ & 400 & Low frequency \\
$\mathrm{f}_{\text {high }}(\mathrm{MHz})$ & 1500 & High frequency \\
Delay Step $(\mathrm{ps})$ & 20 & time delay step \\
Number of delay steps & 64 & number of delay steps \\
Extra delay per beam $(\mathrm{ps})$ & 1200 & delay after beamformed \\
Number of beams formed & 2 & 2 independent beams \\
Number of input Channels & 4 & 4 differential inputs \\
Phase errors & $\pm 10^{\circ}$ & within 10 degrees \\
Total delay (ns) & 2.4 & 1.2 ns extra delay on each beam \\
System gain $(\mathrm{dB})$ & 18 & Amplitude \\
number of gain steps & 16 & 4 bits \\
gain change per step $(\mathrm{dB})$ & 0.5 & Amplitude \\
Power consumption $(\mathrm{mW})$ & 720 & Power consumption \\
\hline
\end{tabular}

$50 \mu \mathrm{m}$. Polystyrene foams have been used to fill the space between the active layer and the ground plane, and the space between the active and the passive layers. The C-ORA finite array has been measured in a Compact Antenna Test Range (CATR). The size of the CATR is $25.1 \mathrm{~m}(\mathrm{~L}) \times 14 \mathrm{~m}(\mathrm{~W})$. The total path distance from the transmit antenna to the antenna array under test is approximately $33 \mathrm{~m}$ by using a subreflector. Directional EMC Test-Antenna HyperLOG 3080 from Aaronia AG is used as the transmit antenna for the C-ORA array radiation pattern measurements.

The LNA and beamformer MMIC chips for the C-ORA prototype array are based on NXP QUBiC4Xi $0.25 \mu \mathrm{m}$ BiCMOS technology. NXP has developed a carbondoped $\mathrm{SiGe}(\mathrm{SiGe}: \mathrm{C}) \mathrm{BiCMOS}$ process (called QUBiC4xi) that has transformed the traditional, high-volume silicon processes. It offers improved $\mathrm{F}_{t}(\geq 200 \mathrm{GHz})$ with $1.4 \mathrm{~V}$ breakdown voltage and a potentially lower noise figure $(\mathrm{NF} \leqslant 0.5 \mathrm{~dB}$ at 10 $\mathrm{GHz})$. The LNA PCB is a four layered design with Isola I-Tera substrate $\left(\epsilon_{r}=3.45\right.$, $\left.\tan _{d}=0.0031\right)$.

The LPDA elements are been designed for mass production so that they can be fabricated using commercial techniques. The metal parts will be likely made out of steel (either mild steel with a protective coating or galvanized steel). The arms of the LPDA can be fabricated from sheet metal using a press tool. These arms will be flat thin sheets (approx. 1mm thick) and will be welded to off the shelf square metal tubes that form the transmission line of the antenna (commonly known as the boom of the LPDA). The different dipoles forming the antenna will also be made as light as possible by taking advantage of the fact that the electric currents will mostly flow in the outer edges of the dipoles and therefore most of the metal in the central regions can be safely removed without a significant impact on performance. The support parts for the antenna will be molded for mass production and will help support the antenna from the metal ground plane. We envisage this to be an elevated ground plane 


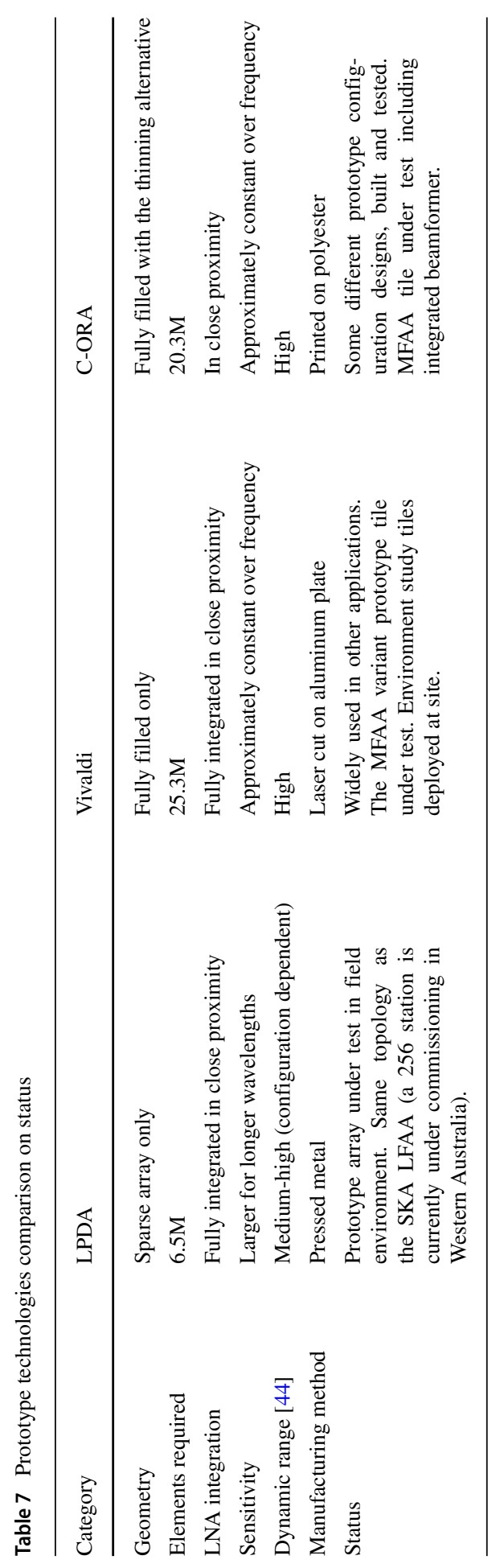


to allow for an easy deployment and maintenance process. Furthermore the LNA (1 per polarization) will be hosted inside the square tube forming the transmission line, at the top, near the feeding point of the antenna. The LNA needs to be fully enclosed inside the tube in order to function without the need of a balun. This also allows for a weatherproof structure that won't need any extra protection (eg. a radome) making the mechanical construction durable and affordable. Finally, the signals from all the antennas in a tile will be collected into a metal box underneath the ground plane where signal conditioning, power feeding to the LNAs and transformation to RF over Fiber for long distance data transmission will take place.

\section{Front-end technology discussions}

Front-end technologies play a significant role on the MFAA system design and performance. A critical factor for array design consideration is the receiver noise, which is dominated by the front-end components. Several prototypes have been built and tested by different institutions using different technologies. Each demonstrates its unique characteristics. A summary of advantages and disadvantages of different front-end technologies is given in Table 7.

\section{Conclusion}

This paper provides the science community with a recent development summary on MFAA front-end design of SKA. The collaboration study on the front-end design have shown several possible routes to deliver the MFAA telescope for SKA science observations. The array antenna selection and optimum antenna spacing array geometry configuration, are the key factors for consideration. Both sparse and dense aperture array configuration with the corresponding antenna prototypes have been investigated. The sparse array geometry demonstrated its advantage to meet the sensitivity requirement by using less number of elements compared to a dense array. However, dense array yields approximately a constant sensitivity across the frequency band and by its nature allows excellent control of sidelobes over wide FoV. Two candidate antennas for dense arrays including C-ORA and Vivaldi antennas have been investigated, C-ORA using a fully differential front-end configuration and Vivaldi with a single-ended architect. The optimum MFAA front-end design can not be simply selected by comparing the performance of the prototypes. It is heavily dependent on distribution of required sensitivity over frequency for prioritized science experiments, availability of subcomponents such as LNA and the affordability on cost and power. Overall MFAA technology development has been continuously progressing over the last decade. Subsystem trade-off optimization activities also see significant improvements in all major challenging areas. The option of using irregular sparse aperture arrays of wide band elements (eg. LPDAs) should be able to leverage knowledge from the developments for the SKA1-LOW (SKA LFAA in phase 1) instrument (an all digital system where the signals from every antenna will be digitalized without prior analogue combination). This concept 
may bring further advantages in terms of extra sensitivity at the low part of the frequency band while reducing the number of antennas and receiving chains by a factor of approximately 4, compared to the dense array. The more demanding processing of high sidelobes in the sparse array is a concern from the dynamic range perspective. This may be addressed with randomization of station geometry within an interferometer array and new calibration techniques. Regardless of the geometry chosen an all digital system will make easier the calibration of the arrays, in particular important for sparse arrays. Progressively technology development is reducing the cost discrepancy between the sparse and dense array. It is envisaged that MFAA technology will become cost effective both for construction and operation by 2025 to continue the exponential performance increase over time in radio telescope science.

Acknowledgements The research and developments in The University of Manchester and University of Cambridge were supported by STFC, UK in the framework of the SKA MFAA project. The authors thank Leonardo MW Ltd for conducting the pattern measurements and Trackwise Designs Ltd for manufacturing the C-ORA array.

Open Access This article is distributed under the terms of the Creative Commons Attribution 4.0 International License (http://creativecommons.org/licenses/by/4.0/), which permits unrestricted use, distribution, and reproduction in any medium, provided you give appropriate credit to the original author(s) and the source, provide a link to the Creative Commons license, and indicate if changes were made.

\section{References}

1. Wilkinson, P.N.: Radio interferometry: Theory, techniques, and applications. In: Proceedings of the 131st IAU Colloquium, Socorro, NM, Oct. 8-12, 1990, vol. 19, p. 428 (1991)

2. Noordam, J.E.: Resolving the sky-radio interferometry: Past, present and future (ISSN 978-1-90920426-3), pp. 79-85 (2012)

3. Swarup, G.: Curr. Sci. 60, 106 (1991)

4. Swarup, G.: Giant metrewave radio telescope (GMRT) - Scientific objectives and design aspects. Indian J. Radio Space Phys. (ISSN 0367-8393) 19, 493 (1990)

5. Parijskij, Y.N.: Radio astronomy of the next century. Astron. Astrophys. Trans. 2(85), 1 (1992)

6. van Ardenne, A., Bregman, J.D., van Cappellen, W.A., Kant, G.W., de Vaate, J.G.B.: Proc. IEEE 97(8), 1531 (2009). https://doi.org/10.1109/JPROC.2009.2021594

7. Taylor, A.R., Braun, R.: Science with the Square Kilometer Array, (ASTRON/NFRA Dwingeloo : Netherlands Foundation for Research in Astronomy) (1999)

8. Carilli, C., Rawlings, S.: Astron. Rev. Elsevier, 48 (2004)

9. Staveley-Smith, L., Oosterloo, T.: Advancing Astrophysics with the Square Kilometre Array (2015)

10. Taylor, A.R.: Concepts for a Large-N Ska, (ASTRON Dwingeloo: Netherlands Foundation for Research in Astronomy) (1999)

11. Hewish, A., Bell, S.J., Scott, P., Collins, R.A.: Observation of a Rapidly Pulsating Radio Source. Nature 48, 709 (1968)

12. Burgary, M. et al.: An increased estimate of the merger rate of double neutron stars from observations of a highly relativistic system. Nature 426, 531 (2003)

13. Bell, J.: Studying pulsars with the SKA and other new facilities. Pulsar astronomy -2000 and beyond. IAU Colloquium 177(48), 711 (2000)

14. Abbott, B.P. et al.: Observation of Gravitational Waves from a Binary Black Hole Merger. Phys. Rev. Lett. 116, 061102(1) (2016)

15. Smits, R., Kramer, M., Stappers, B., Lorimer, D.R., Cordes, J., Faulkner, A.: A\&A 493(3), 1161 (2009). https://doi.org/10.1051/0004-6361:200810383

16. Lazio, T.J.W.: Class. Quant. Grav. 30(22), 224011 (2013). http://stacks.iop.org/0264-9381/30/i=22/ $\mathrm{a}=224011$ 
17. Wilkinson, P.N., Kellermann, K.I., Ekers, R.D., Cordes, J.M., Joseph, T., Lazio, W.: The Exploration of the Unknown. New Astron. Rev. 48, 1551 (2004)

18. van Ardenne, A., Faulkner, A.J., bij de Vaate, J.G.: The square kilometre array: Paving the way for the new 21st century radio astronomy paradigm. Astrophy. Space Sci. Proc., 9-15 (2012)

19. Fender, R., Stewart, A., Macquart, J.P., Donnarumma, I., Murphy, T., Deller, A., Paragi, Z., Chatterjee, S.: Advancing astrophysics with the square kilometre array (AASKA14). Provided by the SAO/NASA Astrophysics Data System, 51 (2015)

20. Smolders, A.B., Kant, G.W.: In: IEEE Antennas and Propagation Society International Symposium. Transmitting Waves of Progress to the Next Millennium. 2000 Digest. Held in conjunction with: USNC/URSI National Radio Science Meeting, vol. 1, pp. 162-165. https://doi.org/10.1109/APS. 2000.873735 (2000)

21. Gunst, A.W., van Haarlem, M.P., Vermeulen, R.C.: In: 2011 XXXth URSI General Assembly and Scientific Symposium, pp. 1-1. https://doi.org/10.1109/URSIGASS.2011.6051279 (2011)

22. Kant, G.W., Patel, P.D., Wijnholds, S.J., Ruiter, M., van der Wal, E.: IEEE Trans. Antennas Propag. 59(6), 1990 (2011). https://doi.org/10.1109/TAP.2011.2122233

23. Lonsdale, C.J. et al.: The Murchison Widefield Array: Design Overview. Proc. IEEE 97(8), 1497 (2009)

24. de Lera Acedo, E., Faulkner, A.J., bij de Vaate, J.G.: In: 2016 United States National Committee of URSI National Radio Science Meeting (USNC-URSI NRSM) (2016), pp. 1-2. https://doi.org/10.1109/USNC-URSI-NRSM.2016.7436228

25. Torchinsky, S.A., Broderick, J.W., Gunst, A., Faulkner, A.J.: SKA document number: SKA-TELMFAA-0200009 (2016)

26. Hall, P.J.: The square kilometre array: An engineering perspective. Springer, ISBN 1-4020-3797-x, 1-4020-3798-8 (2005)

27. van Cappellen, W.A. et al.: ArXiv instrumentation and methods for astrophysics. arXiv:1612.07917 (2016)

28. Lewis, L., Fassett, M., Hunt, J.: In: 1974 Antennas and Propagation Society International Symposium, vol. 12, pp. 335-337. https://doi.org/10.1109/APS.1974.1147206 (1974)

29. Gibson, P.J.: In: 1979 9th European Microwave Conference, pp. 101-105. https://doi.org/10.1109/ EUMA.1979.332681 (1979)

30. Shin, J., Schaubert, D.H.: A parameter study of stripline-fed Vivaldi notch-antenna arrays. IEEE Trans. Antennas Propag. 47(5), 879 (1999). https://doi.org/10.1109/8.774151

31. Wijnholds, S., Kant, G.W., van der Wal, E., Benthem, P., Ruiter, M., Picard, P., Torchinsky, S., Montebugnoli, S. In: Torchinsky, S.A. et al. (eds.) Proc. Wide Field Science and Technology for the SKA, p. 259, Limelette (2009). ISBN 978-90-805434-5-4

32. Olofsson, H. In: Torchinsky, S.A. et al. (eds.) Proc. Wide Field Science and Technology for the SKA, p. 253, Limelette (2009). ISBN 978-90-805434-5-4

33. Benthem, P., Kant, G.W.: In: 2012 6th European Conference on Antennas and Propagation (EUCAP), pp. 629-633. https://doi.org/10.1109/EuCAP.2012.6206634 (2012)

34. Torchinsky, S., Olofsson, A., Censier, B., Karastergiou, A., Serylak, M., Renaud, P., Taffoureau, C.: J. Instrum. 10(07), C07002 (2015). http://stacks.iop.org/1748-0221/10/i=07/a=C07002

35. Torchinsky, S., Olofsson, A., Censier, B., Karastergiou, A., Serylak, M., Picard, P., Renaud, P., Taffoureau, C.: A\&A 589, A77 (2016). https://doi.org/10.1051/0004-6361/201526706

36. van Cappellen, W.A., Bakker, L.: In: 2010 IEEE International Symposium on Phased Array Systems and Technology, pp. 640-647. https://doi.org/10.1109/ARRAY.2010.5613297 (2010)

37. Woestenburg, E.E.M., Witvers, R.H., Ruiter, M., Benthem, P.: In: 2014 International Conference on Electromagnetics in Advanced Applications (ICEAA), pp. 147-150. https://doi.org/10.1109/ICEAA. 2014.6903844 (2014)

38. Ruiter, M., van Cappellen, W., van der Wal, E., Arts, M., van den Brink, R., Visser, K.: In: 2016 10th European Conference on Antennas and Propagation (EuCAP), pp. 1-4. https://doi.org/10.1109/ EuCAP.2016.7481742 (2016)

39. Zhang, Y., Brown, A.K.: Octagonal Ring Antenna for a Compact Dual-Polarized Aperture Array. IEEE Trans. Antennas Propag. 59(10), 3927 (2011). https://doi.org/10.1109/TAP.2011.2163742

40. Lintignat, J., Grima, M., Darfeuille, S., Barelaud, B., Jarry, B., Barth, S., Meunier, P., Gamand, P.: In: SKADS, ASTRON. The Netherlands, SKADS Memo T12. [Online]. Available: http://www.skads-eu. org/ (2008) 
41. de Lera Acedo, E., Razavi-Ghods, N., Troop, N., Drought, N., Faulkner, A.J.: SKALA, a log-periodic array antenna for the SKA-low instrument: design, simulations, tests and system considerations. Exp. Astron. 39(3), 567 (2015)

42. Colin-Beltran, E., Faulkner, A.J., de Lera-Acedo, E.: In: 2014 International Conference on Electromagnetics in Advanced Applications (ICEAA), pp. 746-749. https://doi.org/10.1109/ICEAA.2014. 6903955 (2014)

43. Abraham, J., Colin-Beltran, E., de Lera Acedo, E., Faulkner, A.J.: In: 2016 10th European Conference on Antennas and Propagation (EuCAP), pp. 1-4. https://doi.org/10.1109/EuCAP.2016.7481792 (2016)

44. Braun, R., van Cappellen, W.: SKA memo, 87 (2006)

45. Schilizzi, R.T., Alexander, P., Cordes, J.M., Dewdney, P.E., Ekers, R.D., Faulkner, A.J., Gaensler, B.M., Hall, P.J., Jonas, J.L., Kellermann, K.I.: SKA memo 100, 22 (2007)

46. Medellín, G.C.: SKA Memo 95: Antenna Noise Temperature Calculation. US-SKA tech memo series. Memo 95, 1-12 (2007)

47. El-Makadema, A., Zhang, Y., Yang, M., Brown, A.K.: In: 2015 International conference on electromagnetics in advanced applications (ICEAA), pp. 533-536. https://doi.org/10.1109/ICEAA.2015. $7297172(2015)$

48. Woestenburg, E.E.M., Kuenen, J.C.: Low Noise Performance Perspectives Of Wideband Aperture Phased Arrays. Exper. Astron. 17(1), 89 (2004). https://doi.org/10.1007/s10686-005-6382-5

49. Belostotski, L., Haslett, J.W.: IEEE J. Solid State Circ. 42(11), 2492 (2007)

50. Bhaumik, S. In: Torchinsky, S.A. et al. (eds.) Proc. Wide Field Science and Technology for the SKA, pp. 279-283, Limelette (2009). ISBN 978-90-805434-5-4

51. Weinreb, S., Bardin, J., Mani, H., Jones, G.: Matched wideband low-noise amplifiers for radio astronomy. Rev. Sci. Instrum. 80(4), 044702 (2009). https://doi.org/10.1063/1.3103939

52. Zhang, Y., Brown, A., Yang, M., El-Makadema, A., Harison, S.F., Bosse, S.: In: 2015 International Conference on Electromagnetics in Advanced Applications (ICEAA), pp. 291-292. https://doi.org/10.1109/ICEAA.2015.7297120 (2015)

53. van Noort, W.D., Rodriguez, A., Sun, H., Zaato, F., Zhang, N., Nesheiwat, T., Neuilly, F., Melai, J., Hijzen, E.: In: 2008 IEEE Bipolar/BiCMOS Circuits and Technology Meeting, pp. 93-96 (2008)

54. Belostotski, L., Haslett, J.W.: A Technique for Differential Noise Figure Measurement of Differential LNAs. IEEE Trans. Instrum. Meas. 57(7), 1298 (2008). https://doi.org/10.1109/TIM.2008.917673

55. Bardin, J.C., Weinreb, S.: A 0.1-5 GHz Cryogenic SiGe MMIC LNA. IEEE Microwave Wireless Comp. Lett. 19(6), 407 (2009)

56. de Vaate, J.G.B., Bakker, L., Woestenburg, E.E.M., Witvers, R.H., Kant, G.W., van Cappellen, W.: In: 2009 13th International Symposium on Antenna Technology and Applied Electromagnetics and the Canadian Radio Science Meeting, pp. 1-4 (2009)

57. Krüger, P., Visser, B., Prinsloo, D.: In: 2017 47th European Microwave Conference (EuMC), pp. 58-61. https://doi.org/10.23919/EuMC.2017.8230798 (2017)

58. Weinreb, S.: Very Low Noise Ambient-Temperature Amplifiers for the 0.6 to $1.6 \mathrm{GHz}$ Range. SKA memo 137, 1-7 (2011)

59. Abraham, J., Van, H.B., de Lera Acedo, E., Craeye, C.: In: 2017 11th European Conference on Antennas and Propagation (EUCAP), pp. 3837-3841. https://doi.org/10.23919/EuCAP.2017.7928253 (2017)

60. Shaw, R.D., Hay, S.G., Ranga, Y.: In: 2012 International Conference on Electromagnetics in Advanced Applications, pp. 438-441 (2012)

61. Ranga, Y., Shaw, R.D., Hay, S.G.: In: The 8th European Conference on Antennas and Propagation (EuCAP 2014), pp. 1860-1864 (2014)

62. Morawietz, J., Witvers, R.H., de Vaate, J.G.B., Woestenburg, E.E.M.: In: 2007 European Radar Conference, pp. 291-294 (2007)

63. Garcia-Perez, O., Segovia-Vargas, D., Garcia-Munoz, L.E., Jimenez-Martin, J.L., Gonzalez-Posadas, V.: Broadband Differential Low-Noise Amplifier for Active Differential Arrays. IEEE Trans. Microwave Theory Techniq. 59(1), 108 (2011)

64. Arts, M., Maaskant, R., de Lera Acedo, E., bij de Vaate, J.G.: In: 2009 3rd European Conference on Antennas and Propagation, pp. 566-570 (2009)

65. Zhang, Y., Brown, A.K., Yang, M., El-Makadema, A.: In: 2017 11th European conference on antennas and propagation (EUCAP), pp. 3850-3853. https://doi.org/10.23919/EuCAP.2017.7928738 (2017) 